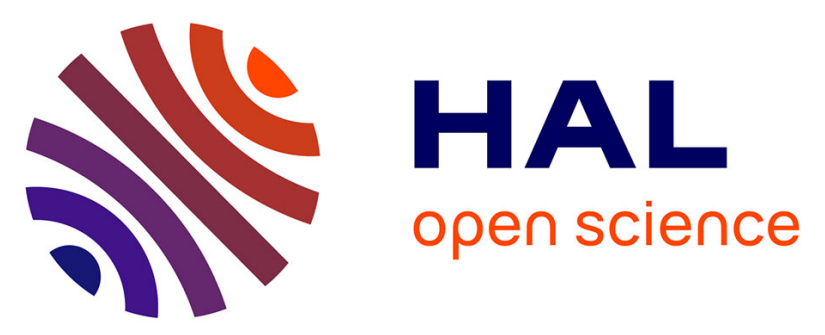

\title{
Ce que peut apporter la résilience à la prévention des désastres. Exemples en Lavours et en Chautagne (Ain, Savoie)
}

Patrick Pigeon, Julien Rebotier, Bernard Guézo

\section{> To cite this version:}

Patrick Pigeon, Julien Rebotier, Bernard Guézo. Ce que peut apporter la résilience à la prévention des désastres. Exemples en Lavours et en Chautagne (Ain, Savoie). Annales de géographie, 2018, 1 (719), pp.5-28. halshs-01702344

\section{HAL Id: halshs-01702344 \\ https://shs.hal.science/halshs-01702344}

Submitted on 6 Feb 2018

HAL is a multi-disciplinary open access archive for the deposit and dissemination of scientific research documents, whether they are published or not. The documents may come from teaching and research institutions in France or abroad, or from public or private research centers.
L'archive ouverte pluridisciplinaire HAL, est destinée au dépôt et à la diffusion de documents scientifiques de niveau recherche, publiés ou non, émanant des établissements d'enseignement et de recherche français ou étrangers, des laboratoires publics ou privés. 
Pigeon Patrick, Rebotier Julien, Guézo Bernard, (2018), « Ce que peut apporter la résilience à la prévention des désastres : Exemples en Lavours et en Chautagne (Ain, Savoie) », Annales de géographie, n719, 1/2018 : 5-28.

Disponible sur : http://www.revues.armand-colin.com/geographie/annalesgeographie/annales-geographie-ndeg719-12018/ce-que-peut-apporterresilience-prevention-desastres-exemples-lavours-chautagne-ain

\section{ARTICLE}

PIGEON Patrick

Professeur des universités

Université de Savoie-Mont-Blanc

Technolac, 73376 Le Bourget-du-Lac CEDEX

patrick.pigeon@univ-smb.fr

\section{REBOTIER Julien}

Chargé de recherche CNRS

LISST-UMR 5193, Université Toulouse Jean Jaurès, Maison de la Recherche, 5 allées Antonio Machado, 31058 Toulouse Cedex 9

julien.rebotier@cnrs.fr

\section{GUEZO Bernard}

Responsable du Groupe Vulnérabilité Urbaine Eau Risques, Cerema

Direction technique Territoires et Ville, 2 rue Antoine Charial, 69426 Lyon Cedex 3

Bernard.Guezo@cerema.fr 


\section{Ce que peut apporter la résilience à la prévention des désastres: Exemples en Lavours et en Chautagne (Ain, Savoie).}

Résumé : L'article justifie l'intérêt et les limites du recours à la notion de résilience, tant pour la recherche que pour la gestion, dans le domaine de la prévention des risques de désastres. Pour ce faire, il mobilise deux sites près du Rhône où l'aléa inondation est comparable (différencié, mais présentant des points communs), l'un à Culoz, près des marais de Lavours, et l'autre en Chautagne, sur la commune de Chanaz, mais où le risque d'inondation est géré de manière opposée. La compréhension du contraste de gestion et de peuplement observé révèle la recomposition de trois approches-types de la prévention qui sont: aléa centrée, approche territorialisée des risques, et résilience. L'intrication de ces approches successives permet de comprendre pourquoi il peut y avoir autant de définitions différentes de la résilience, leur caractère possiblement contradictoire, et les nombreuses contestations de la notion. Mais elle permet aussi de défendre pourquoi une approche intégrée de la résilience est utile à la compréhension des risques de désastres, tout autant qu'à la gestion. Ainsi envisagée, la résilience permet d'expliciter les apports, en même temps que les limites, des politiques de prévention des risques de désastres.

Mots-clés : résilience, désastre, risque, aléa, politiques de prévention, territoire, inondation, digue, plans de prévention des risques, France

What Can Resilience Bring to Disaster Prevention: Examples Taken in Lavours and Chautagne (Ain, Savoie - France). 
Abstract: This paper sheds light on how the notion of resilience, in spite of its limitations, may contribute to a better understanding and management of disaster risk. The method used draws on two sites near to the Rhone, where flood hazard is similar to some extent. The first one is located in Culoz municipality, close to Lavours Swamps. The other one is situated in Chautagne, in Chanaz municipality. Yet, both cases show opposite risk management choices. Understanding the differences in both risk management and local development makes it possible to identify the reshaping of three major approaches of risk prevention, which are: a hazard-centered approach, a territory-based approach, and, today, a resilience-oriented approach. Considering those successive and intertwined risk approaches helps us to understand why the notion of resilience is so polysemic, why it potentially bears in itself so many contradictions and why it is questioned so much. Yet, drawing on the same elements, it is possible to defend why an integrated approach of resilience might be useful in understanding and managing risk. It could make both the benefits and shortcomings of disaster risk prevention policies more visible and explicit.

Keywords: resilience, disaster, risk, hazard, prevention policies, territory, floods, dike, risk prevention plans, France

La notion de résilience est toujours plus mobilisée dans la recherche sur les risques de désastres et leur prévention depuis le début du 21ème siècle. Des publications soulignent pourtant son caractère polysémique, une faiblesse si cette polysémie n'est pas précisée (Birkmann, 2006; Reghezza-Zitt et Rufat, 2015a). Elles questionnent l'intérêt de la résilience pour la gestion et la prévention des risques (Guézo et Pigeon, 2015; Brun et Gache, 2013 ; UNISDR, 2015). Divers conditionnements de cette notion sont aussi abordés (Alexander, 2013 ; Evans \& Reid, 2014). Ces différents éléments 
sont synthétisés dans plusieurs ouvrages, comme le rapport global sur la prévention des désastres que publie l'UNISDR (2015).

Ces nombreuses réserves justifient l'article : comment comprendre l'engouement pour la résilience alors que cette notion est si difficile à définir, si limitée, et autant conditionnée ? Les limites de la gestion et de l'interprétation fondées sur l'approche aléa centrée des risques de désastres sont connues. Ces limites expliquent l'engouement des dernières années pour la territorialisation des risques, notamment l'attention portée aux échelles locales pour la mise en place des politiques de prévention, et à leur inscription dans des contextes socio-spatiaux spécifiques (Donze, 2007). Mais à son tour, cette tendance n'a pu satisfaire toutes les attentes, notamment celles exprimées en matière de reconnaissance des risques dans leur globalité, et de mise en partage de leurs modes de gestion. La résilience se diffuse plus récemment, comme tentative de réponse à ces aspirations. Dans de tels entrecroisements des approches, quel peut être son apport original pour la compréhension et pour l'action?

Nous proposons des réponses à ces questions en nous appuyant sur une étude de cas, résultat d'une réflexion conjointe entre les trois auteurs. Deux d'entre eux sont plus académiques, avec des terrains de recherche différents (Amérique latine ou Europe). Le troisième est plus sur le versant gestionnaire. Il travaille principalement en France, pour le Cerema, tout en ayant des expériences internationales (Indonésie). Certes, l'étude de cas est le reflet de contextes locaux. Mais elle peut également recouper des questions qui se posent dans d'autres contextes culturels, économiques, politiques, et qui concernent la thématique de la prévention des désastres. Ainsi, une étude de cas peut illustrer des réponses aux questions posées qui combinent le contexte local et des éléments qui le transcendent.

Le terrain qui nous aide à discuter de l'intérêt de la notion de résilience est situé dans la partie médiane du bassin-versant du Rhône, dans les zones humides associées au lit majeur du Rhône, sur la commune de Culoz, et en 
Chautagne, dans les départements de l'Ain et de la Savoie. Sur deux sites communaux confrontés à l'aléa inondation de manière comparable malgré des différences que nous préciserons sur la base de contacts pris à la DREAL, le traitement institutionnel des risques associés s'avère opposé. Dans le premier cas, la commune de Culoz (Ain) n'a pas pu réaliser un port de plaisance et sa marina, notamment en raison du Plan de prévention des risques (PPR). Les terrains concernés par le projet y apparaissent en zone rouge, non constructible, malgré la présence de plusieurs digues, dont l'une est gérée par la Compagnie Nationale du Rhône (CNR). Dans le second cas, celui de la commune de Chanaz (Savoie), le port de plaisance ainsi que des logements ont pu voir le jour, malgré la reconnaissance officielle du risque d'inondation. Certains de ces logements se trouvent même en zone rouge du PPR.

Ces différences dans la gestion du risque associé aux inondations pour des sites confrontés à des aléas qui demeurent comparables malgré des différences d'aménagement entre les deux berges du Rhône, fondent un raisonnement qui discute des apports et des limites de la notion de résilience. Comprendre le décalage entre les deux communes impose de prendre en compte la diversité des acteurs agissant à plusieurs échelles en fonction des arbitrages qu'ils opèrent, mais aussi des ressources disponibles pour organiser le territoire en tenant compte de l'aléa inondation. Les contradictions visibles tant sur les plans d'aménagement que sur les terrains ne peuvent être expliquées que partiellement à partir de l'échelle locale.

C'est donc à l'aune de cette étude de cas que l'on se demande ce que peut apporter la notion de résilience par rapport à une simple lecture multiscalaire, classique en géographie, ou par rapport à une approche très centrée sur la dimension politique et les rapports de force asymétriques entre acteurs, comme il peut en être question dans une approche territorialisée des risques. Les expressions d'emboîtement d'échelles et de jeux d'acteurs ne sont-elles pas trop descriptives et statiques pour pouvoir répondre aux 
besoins académiques et aux attentes des gestionnaires confrontés à la prévention des désastres? Ne s'inscrivent-elles pas trop dans la seule dénonciation, aux dépens de la proposition plus pragmatique qui permet le passage effectif à l'action?

L'intérêt de la notion de résilience se justifierait ainsi à double titre. Elle permettrait de faire plus explicitement apparaître les limites des différentes politiques menées, de reconnaître ces limites, puis de les incorporer, par exemple sous forme d'affichage, au sein des politiques existantes. La résilience inviterait en outre à plus expliciter des enjeux autres que ceux qui sont liés à un seul type de risque (ici, l'inondation), facilitant la prise en compte de la multiplicité des risques et de la concurrence des enjeux (Pigeon, 2012a). Le recours à la résilience relève donc d'un registre heuristique et gestionnaire: la notion permettrait de comprendre les multiples raisons pour lesquelles les politiques de prévention des désastres sont nécessaires, tout en étant nécessairement limitées (Pigeon et Rebotier, 2016). La comparaison des deux terrains permet d'envisager cette possibilité.

Nous défendons ici le fait que la notion de résilience correspond à la recherche de solutions pragmatiques et partagées, à la fois nécessaires et nécessairement limitées, opératoires et précaires. Une telle maniabilité justifie l'engouement pour la notion de résilience, mais aussi le fait qu'elle soit contestée. La résilience envisagée en tant que processus nous paraît cependant plus adaptée à la prévention des risques de désastres que l'approche préexistante, encore trop statique et polarisée par les rapports de force, qui est celle de l'approche territorialisée. En outre, la notion de résilience semble marquer un changement de paradigme pour une gestion des risques qui prendrait explicitement ses distances avec les cultes modernes de la maîtrise et de la certitude (Reghezza, 2015). En l'occurrence, il s'agit d'envisager la résilience dans sa capacité à caractériser a priori les dynamiques et les formes du territoire qui permettent de contenir, 
d'absorber voire de tirer parti d'événements adverses, sans jamais parvenir à les éviter complètement.

Afin d'étayer cette réflexion sur les apports et les limites de la résilience, nous procédons en quatre étapes. Une première partie fait état des deux terrains, exposés de manière voisine à l'aléa inondation, mais opposés dans leur gestion du risque. Des facteurs locaux, propres à chacun des territoires, viennent ensuite éclairer ces différences entre les communes; avant de prendre en compte les acteurs et leurs intérêts aux échelles régionale et nationale. Dans une quatrième partie, nous discutons des apports et des limites spécifiques de la résilience pour la compréhension et la gestion des risques, en regard d'autres approches géographiques, dont l'approche territorialisée des risques. Cette partie approfondit le cas des constructions en zone rouge dans la commune de Chanaz, exemple possible, et discuté, de résilience appliquée.

\section{Culoz et Chanaz : Deux communes confrontées à un aléa hydraulique qui reste comparable, mais très différentes dans leur relation au fleuve}

\subsection{Deux sites confrontés de façon comparable à un aléa hydraulique}

Des caractéristiques similaires valent aux communes de Culoz et de Chanaz d'être concernées par les risques liés aux inondations du Rhône. Les communes recouvrent partiellement les marais de Chautagne et de Lavours, liés à une ancienne frontière entre la France et la Savoie, jusqu'en 1860 (Bravard, 1986). Ces zones humides sont des aires d'expansion des crues du Rhône.

Ces communes ont également été concernées par les travaux de rectification du lit mineur du Rhône. La CNR y a renforcé l'endiguement du fleuve à l'occasion des travaux liés aux équipements hydroélectriques. La commune de Culoz est à l'aval du canal de fuite de l'usine hydroélectrique de Chautagne, tandis que la commune de Chanaz se trouve immédiatement à l'amont du canal d'amenée à l'usine de Belley (Figure 1). Le canal 
d'amenée désigne la partie totalement artificialisée du Rhône qui voit passer la part du débit du fleuve qui sera turbinée.

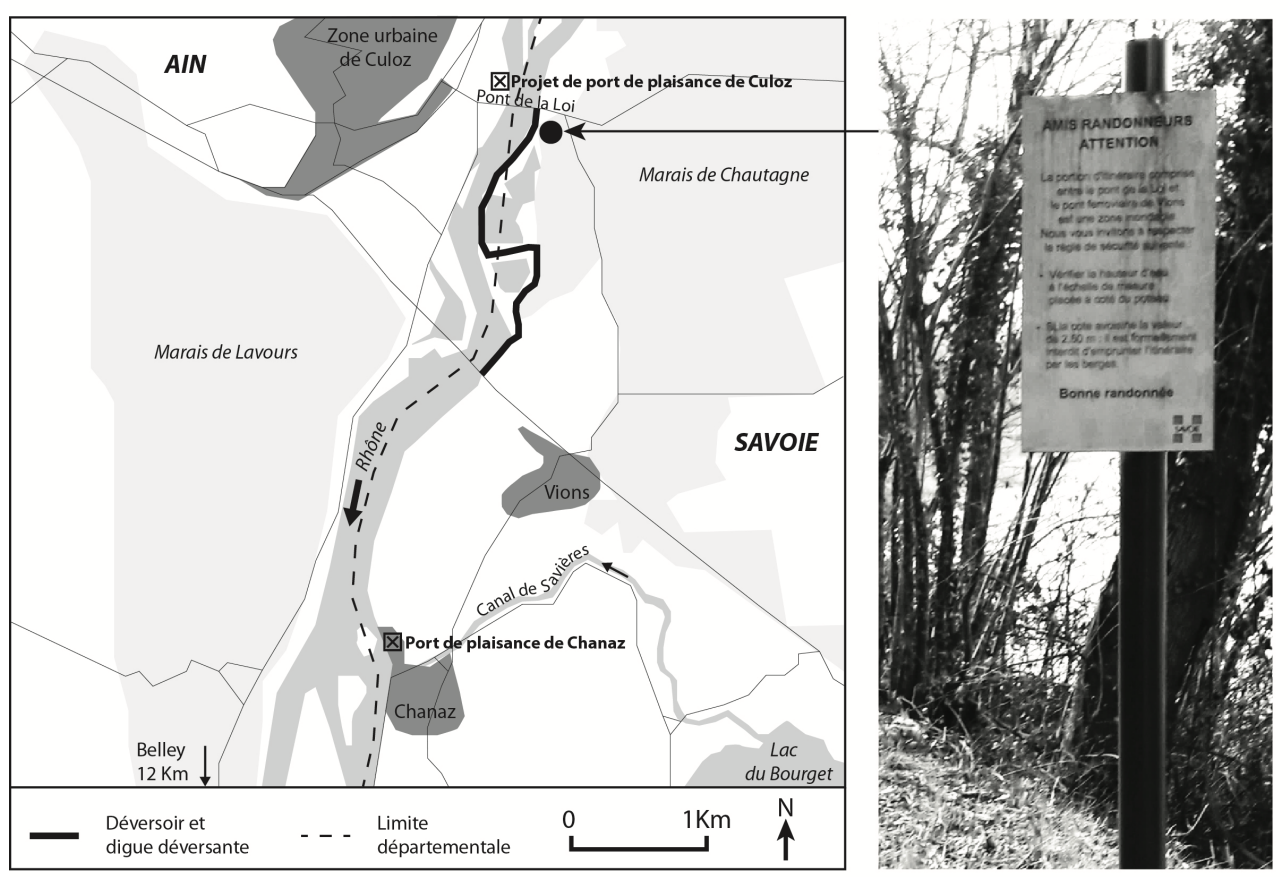

Figure 1. Carte de localisation des communes de Chanaz et Culoz.

Le panneau pris en photo annonce le caractère submersible des digues en rive gauche du Rhône, immédiatement au sud du pont de la Loi (carte d'après Trapatel et Fruchart, 2012 ; photo : février 2017).

\section{Figure 1. Location map of Chanaz and Culoz municipalities.}

The picture shows a board stating that the dikes on the left bank of the river Rhône are liable to flooding, just south to the pont de la Loi bridge (map taken from Trapatel et Fruchart, 2012; photo: February 2017).

Il existe cependant des différences de fonctionnement hydraulique entre les deux sites, comme le démontrent Klingeman et alii (1994) : elles peuvent varier même sur une distance courte. L'une de ces différences est liée à 
l'existence de digues déversoirs, entre le pont de la Loi au nord et le pont de Vions au sud, et uniquement sur la berge savoyarde du Rhône. Ces surverses entrent en fonction à partir d'un débit de $700 \mathrm{~m}^{3} / \mathrm{s}$ et permettent à la plaine de Chautagne de conserver, notamment sur la commune de Chanaz, son rôle de plaine d'expansion des crues malgré les aménagements de la CNR. Un panneau informe les promeneurs du danger à circuler sur les berges du Rhône en rive gauche (Figure 1). Cette fonction régulatrice permet d'espérer, en s'appuyant sur les modélisations effectuées avec tests partiels intégrant les débits mesurés pour la crue de référence centennale, que la crue de référence millénale passe de $4150 \mathrm{~m}^{3} / \mathrm{s}$ à l'amont de la plaine de Chautagne à $2800 \mathrm{~m}^{3} / \mathrm{s}$ à l'aval (Trapatel et Fruchart, 2012). Une autre différence provient de la présence en rive droite du Rhône cette fois du Seran, rejoignant le fleuve au sud de la commune de Culoz. L'aménagement du Rhône a nécessité de maintenir cet exutoire par la réalisation d'un ouvrage en siphon. L'inondation sur la commune de Culoz peut provenir des débordements du cours d'eau, mais aussi du fonctionnement inversé de cet ouvrage lors d'une crue du Rhône.

Néanmoins, ces différences de fonctionnement hydraulique ne changent pas fondamentalement l'inondabilité des lieux. Les deux communes sont confrontées à l'aléa d'inondation, qui reste comparable, et leur PPR le reconnaît officiellement. Les terrains appartenant à la commune de Culoz, et que nous étudions ici, se trouvent juste à l'aval de la confluence entre le Rhône court-circuité et le canal de fuite de l'aménagement hydroélectrique de Chautagne (usine d'Anglefort). Les digues qui les bordent contiennent les débits du Rhône en période normale. La note de présentation du PPR de Chautagne (2015 : 17) relève ainsi que, depuis les aménagements de la CNR en 1980, « l'intégralité du débit revient en amont de Culoz, ensuite le Rhône longe la plaine de Chautagne à plein débit pendant deux kilomètres environ », ceci pour un débit inférieur à $700 \mathrm{~m}^{3} / \mathrm{s}$, avant que les digues déversoirs au sud du pont de la Loi ne_commencent à être opérationnelles. 
En effet, sur les deux communes, les zonages des PPR reprennent les mêmes principes, nationaux, pour les zones bleues, constructibles sous conditions, et pour les zones rouges, inconstructibles. On rappelle que ces documents sont prescrits par les préfets, représentants de l'Etat dans les départements. Pour ces deux cas, nous observons aussi la prise en compte du risque digue. Ce type de zonage admet officiellement qu'une digue ne permet pas d'éliminer tout risque de dommage (Pigeon, 2014).

Ce descriptif permet de justifier la comparaison de sites appartenant à deux communes confrontées à des aléas d'inondation comparables. Toutefois, l'affichage du risque de désastre lié aux inondations du Rhône est opposé à plusieurs titres.

\subsection{Le risque d'inondation inégalement intégré et affiché}

Sur le PPR de Culoz (2004), les terrains immédiatement à l'arrière des digues CNR sont en zone rouge. Le Plan local d'urbanisme - PLU de Culoz (2009) contient un «plan de servitudes et d'informations » qui reporte ici une zone rouge R1 suivie d'une zone bleue B2, en conformité avec le zonage PPR. La zone bleue B2 est décrite comme une « zone inondable en cas de rupture de digue, constructible sous certaines conditions ». De plus, la carte d'aléa du PPR mentionne plusieurs flèches qui représentent des « submersions par infiltrations » sur le même site. Elles attirent encore plus l'attention sur les limites des digues dans ces secteurs (Figure 2, PLU Culoz, 2009 ; PPR Culoz, 2004). Le travail de terrain vérifie la réalité de ces infiltrations (Figure 2). Deux autres digues portant la route et la voie ferrée Lyon-Genève complètent les travaux de correction/protection. Même si ces digues sont insuffisantes, leur multiplication rend le zonage étonnant, car il souligne avant tout les limites des ouvrages de correction, malgré leur nombre. 

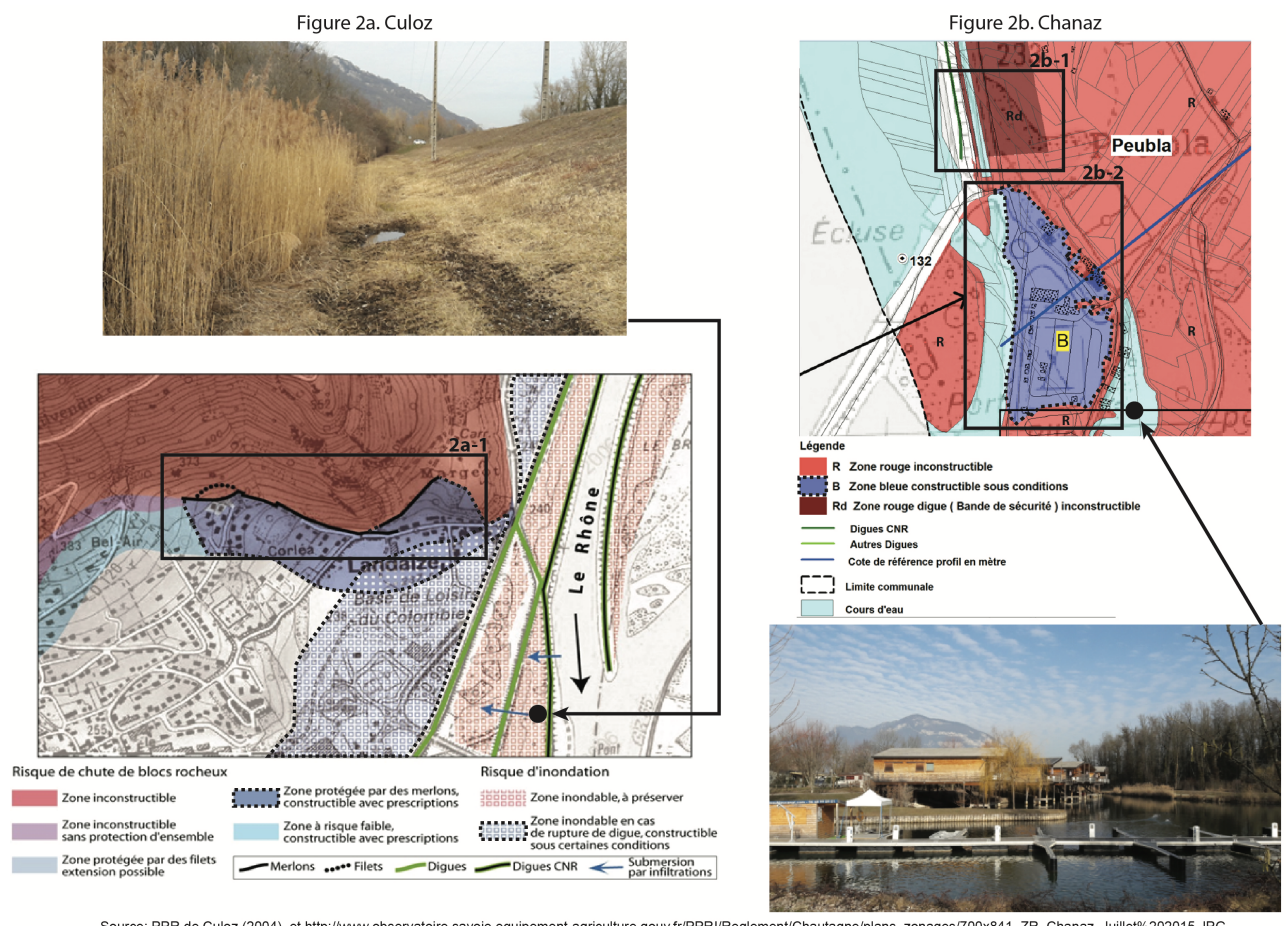

Figure 2. Comparaison des zonages PPR de Culoz et de Chanaz.

2a. Culoz: la photographie vérifie l'existence d'infiltrations notamment sous la digue CNR, justifiant la zone rouge inondation.

2b. Chanaz: la photographie montre l'habitat palafittique en zone rouge du PPR.

L'appel aux rectangles numérotés figure dans le schéma de synthèse.

Figure 2. Comparing risk zoning in both Culoz and Chanaz risk prevention plans.

2a. Culoz: the photography highlights the existence of leaks, in particular under the CNR dike, justifying the red zone for flooding.

2b. Chanaz: the photography shows the buildings on piles, inside the red zone of the risk prevention plan.

The reference to the numbered boxes appears in Figure 4. 
La consultation des archives municipales de Culoz, tout comme un entretien avec un ancien membre du Conseil municipal de Culoz et le directeur des services techniques de la municipalité, permettent de confirmer qu'il a pourtant été envisagé d'utiliser ces terrains pour développer un port de plaisance fluviale. Le rapport de présentation du PLU (2009 : 99) mentionne même que «le projet (...) est une des innovations du PLU de Culoz ». Il en détaille les avantages et les contraintes aux pages 100, 124 et 132 . L'opération d'aménagement aurait permis de tirer parti des terrains qui y ont été exploités sous forme de gravières, désormais épuisées. Egalement, elle est cohérente avec la volonté de «valoriser l'entrée est de l'agglomération sur le site de l'île de Vions » (2009 : 87). Le zonage du PPR actuel a rendu impossible la réalisation de ce projet, même si, comme nous le verrons cidessous, d'autres raisons, plus structurelles, permettent aussi de comprendre son abandon.

Le parallèle avec la commune de Chanaz est instructif. La municipalité a développé un port de plaisance. Son historique peut être reconstitué à partir des registres des délibérations du Conseil municipal. Ici aussi, les travaux de la CNR ont été engagés à partir du début de la décennie 1980, et la compagnie a collaboré à de nombreuses reprises avec la municipalité pour promouvoir un port de plaisance. Pourtant, le site aménagé se trouve juste à l'amont de la confluence entre le canal de Savières et le Rhône, là où ce dernier concentre aussi tout son débit en période normale. Ce n'est qu'à l'aval de la confluence entre le canal de Savières et le Rhône que le fleuve se scinde en deux : le canal d'amenée vers la centrale hydroélectrique de Belley et le Rhône court-circuité (Figure 1).

A l'amont de la confluence entre le canal de Savières et le Rhône, les digues qui bordent le site aménagé en port de plaisance présentent donc une forte similitude avec celles qui concernent la commune de Culoz sur le site du projet de marina, abandonné : elles contiennent tout le débit du Rhône en période normale, c'est-à-dire avant que les digues déversoirs ne soient 
opérationnelles. Le PPR qui concerne la commune de Chanaz fait aussi explicitement ressortir le risque digue par le zonage dédié (Figure 2), malgré la présence de la digue CNR censée résister à l'aléa de référence du PPR. Toutefois, ce type de zonage disparaît au niveau du port de plaisance, qui, pour l'essentiel, se trouve classé en zone bleue. Plus intéressant encore est le classement d'une partie de la zone liée aux équipements du port et au camping municipal en zone rouge, avec la possibilité offerte de poursuivre, certes avec des réserves, l'urbanisation du secteur.

On peut donc prétendre que sur ces deux sites, l'aléa inondation reste comparable, même si la rive savoyarde du Rhône est plus confrontée aux inondations que la rive de l'Ain, en période de crue du Rhône. La présence des digues de la CNR confirme cette proximité, et l'intérêt de la comparaison. Pourtant, l'intégration du risque d'inondation dans le zonage du PPR est différente. La vulnérabilité des deux sites est devenue inégale, en raison de la possibilité donnée de construire un port de plaisance à Chanaz, ce qui a été refusé à Culoz. Il est encore plus remarquable de constater que c'est la rive savoyarde, plus confrontée aux inondations en période de crue, qui porte le port de plaisance et la marina qui l'accompagne. Comment comprendre le décalage observé ? Cette démarche, classique en géographie, fonde un raisonnement qui permet de défendre une approche possible de la résilience tant dans le domaine académique que gestionnaire.

\section{L'importance des héritages de peuplement et de la contextualisation locale dans la gestion des risques}

\subsection{Des réserves foncières et un pôle d'emplois établi à Culoz.}

Culoz dispose, dans son PLU, de terrains situés en zone blanche du PPR, qui peuvent être ouverts à la construction. L'impossibilité de valoriser les terrains par un port de plaisance, comme le verrouillage du foncier par les zones rouges du PPR, handicapent moins la commune de Culoz. 
Ce que confirme l'histoire de la commune de Culoz, marquée par le nœud ferroviaire, au croisement des lignes entre Lyon, Genève et Chambéry, comme par le développement de l'industrie. Les deux activités n'ont pas de lien direct avec le Rhône. Le site internet de la commune attire d'ailleurs l'attention sur la gare, comme aussi sur l'entreprise principale de la commune qu'est la CIAT :

« À l'heure où la désindustrialisation frappe terriblement notre pays, Culoz est fière d'accueillir sur son territoire le plus gros employeur privé de l'Ain. Avec près de 1000 emplois directs, la CIAT fait vivre de très nombreuses familles ».

Le rapport de présentation du PLU (2009 : 41-43) confirme que la CIAT est bien « le pilier de l'économie locale », tandis que la photo de l'entreprise trône dans la salle des délibérations du Conseil municipal (Figure 3). Le rapport ajoute cependant que « la recherche de diversification de l'emploi est un enjeu majeur », et il aborde le projet de port de plaisance à ce titre. Mais le projet reste secondaire, comme cela a pu être confirmé par les services techniques et un représentant du Conseil municipal de Culoz le 19 décembre 2016.
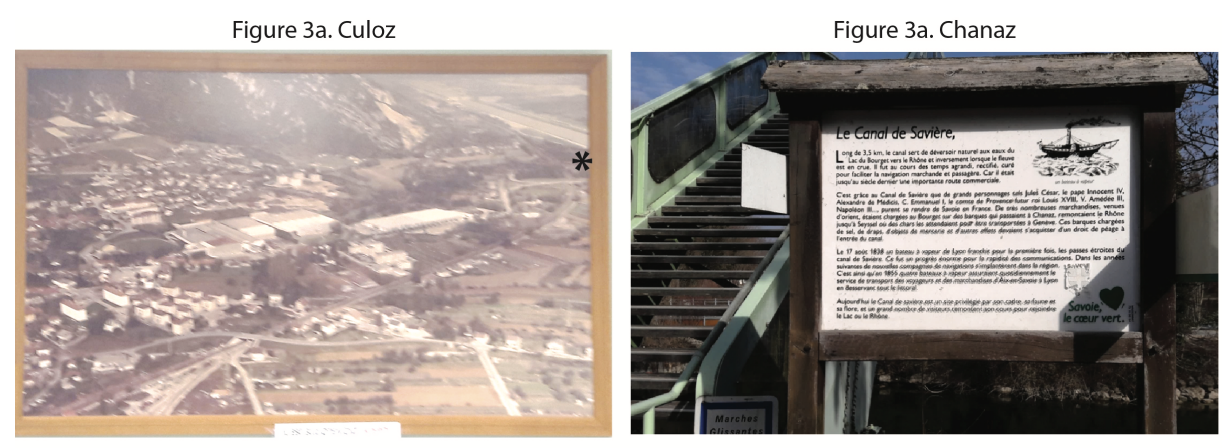

Figure 3 : L'affichage des enjeux, différemment liés au fleuve, à Culoz et à Chanaz

3a. Photo de Culoz représentant la commune, apposée dans la salle des délibérations du Conseil municipal. Le Rhône apparaît en haut et à droite de 
la photo, où se situe le projet de port $\left(^{*}\right)$. La gare et les bâtiments de la CIAT sont au centre.

3b. Panneau installé sur la rive gauche du canal de Savières, montrant l'intérêt de l'enjeu fluvial pour Chanaz, près de la passerelle qui enjambe le canal vers le port de plaisance et l'habitat palafittique, en rive gauche du Rhône.

Figure 3: Putting forward the main stakes, unevenly related to the river Rhône in Culoz and Chanaz

3a. Picture of the municipality of Culoz, shown in the office of the municipal council. The river Rhone appears at the top right corner of the photo, where the project of marina takes place (*). The railway station and CIAT Company building stand at the very centre.

3b. Board on the left bank of the Savières canal, showing the interest of fluvial stakes for Chanaz municipality, close to the footbridge which spans the canal towards the marina and the buildings on piles, on the left bank of the river Rhône.

Pour la commune de Culoz, les marais de Lavours et le Rhône représentent une marge. Qu'ils appartiennent à la zone rouge du PPR est donc moins susceptible de soulever des oppositions, malgré le projet de port de plaisance que ce classement a rendu caduc.

\subsection{Des liens étroits et anciens avec le Rhône et le canal de Savières à}

\section{Chanaz.}

C'est exactement la situation inverse à Chanaz concernant le rapport de la municipalité au Rhône. En effet, les registres des délibérations du Conseil municipal, consultés notamment pour les années 1982 et 1983, font apparaître des dizaines de références aux aménagements des berges du Rhône à la confluence avec le canal de Savières. Il s'agit de mettre en place le port de plaisance fluviale et le « camping-caravaning municipal» associé, 
en s'appuyant sur les aménagements de la CNR. Par exemple, la délibération du 19 novembre 1982 mentionne page 35 : «l'entretien du maire avec la CNR concernant l'aménagement du port de plaisance situé dans le bras d'accès à l'écluse permettant le franchissement du canal de Savières au Rhône. Ces services [de la CNR] seraient prêts à mettre à la disposition de la commune ce chenal d'accès ». Un avant-projet de la direction départementale de l'Equipement évalue l'investissement à 350000 francs HT. En 1983, est inscrite la réalisation de la «troisième tranche du camping quatre étoiles » pour un investissement de 1,2 million de francs. Il s'agit assurément d'un effort budgétaire important pour une commune dont le compte administratif pour 1985 annonce 1,9 million de francs de fonctionnement, et 2,6 millions de francs d'investissement.

L'historique récent de la commune, à partir de 1980, est donc étroitement mêlé au traitement du lien hydraulique entre le canal de Savières et le Rhône. Mais l'ancienneté des relations entre le Rhône et la commune compte aussi. La commune affiche ce passé lointain, qu'elle revendique en lien explicite avec la marina actuelle, sur des panneaux implantés sur les berges du canal, (Figure 3). Le centre historique de la commune se trouve sur la berge sud et gauche du canal.

Le différentiel culturel et de possibilités offertes entre Culoz et Chanaz permet donc de comprendre pourquoi un risque initialement reconnu comme similaire car lié à un aléa comparable au moins en situation normale peut être affiché et intégré de manière aussi contradictoire dans les deux peuplements communaux.

Toutefois, s'il est tentant et classique d'expliquer les différences observées par des particularités locales de peuplement, tout géographe sait que cette démarche est aussi nécessaire qu'insuffisante, notamment ici au regard des principes qui sous-tendent la définition des désastres. Ces derniers sont justifiés par le CRED comme tout événement vérifiant au moins l'un des trois critères : 10 morts, 100 sinistrés, le recours à une aide extérieure à la 
collectivité locale ${ }^{1}$. On peut en déduire qu'un désastre se définit certes par le franchissement d'un seuil d'intensité de dommages, mais qu'il est tout autant associé au dépassement des capacités locales de gestion, justifiant le recours à une échelle supra-locale.

Sur la base de ces principes, le renforcement des capacités locales de gestion et la prise en compte des connaissances locales dans les politiques de prévention comptent parmi les critères que retient la bibliographie sur la prévention des risques de désastres. La recherche de résilience vise elle aussi le renforcement des capacités locales de gestion, sans parvenir pour autant à éviter tout désastre (Birkmann, 2006). Cela étant, la compréhension et la gestion des politiques de prévention des désastres ne peuvent en aucun cas se réduire aux caractéristiques locales des peuplements concernés. C'est d'ailleurs ce qu'atteste la nature étatique du PPR, l'une des entraves à la réalisation du port de plaisance dans la commune de Culoz.

\section{L'inégale prise en compte des enjeux régionaux et nationaux}

\subsection{Le poids des enjeux supra-locaux dans les dispositifs de gestion des} risques

Les zonages du PPR ont pour objectif de prévenir les désastres, principalement en évitant la construction de bâtiments sur des terrains exposés à l'inondation. L'outil de prévention est d'initiative et de prérogative étatiques : il est prescrit par le préfet et résulte du décret d'application du 3 mai 1984, associé à la loi Tazieff. C'est cette dernière, en 1982, qui crée le dispositif actuel de prévention, retouché à de nombreuses reprises (par la loi Barnier, notamment, en 1995). L'outil de prévention à l'échelle locale annonce donc, par sa nature même, la prise en compte d'enjeux d'échelles supra-locale, régionale comme nationale.

$1 \quad$ http://www.emdat.be/guidelines 
C'est particulièrement le cas ici, puisque les marais de Chautagne et de Lavours représentent des zones d'expansion des crues du Rhône. Ces zones contribuent à défendre l'agglomération de Lyon, à l'aval, des crues du Rhône, lesquelles restent associées dans les annales historiques aux deux désastres de 1840 et 1856 (Combes, 2007). Le PLU de Culoz mentionne cette fonction au travers des servitudes d'utilité publique. On lit dans son rapport de présentation que le « service de la navigation Rhône-Saône, sis à Lyon, est responsable de la servitude EL2, défense contre les inondations », tout comme l'est le Ministère de l'équipement au titre du PPR. Enfin, le territoire à risques d'inondations (TRI) de Lyon institué au titre de la mise en œuvre de la directive inondation de 2007 intègre dans son périmètre le Haut-Rhône au titre du principe de solidarité amont-aval. Un des objectifs principaux est de prévenir un futur désastre concernant Lyon en réduisant depuis l'amont le volume et la vitesse de l'onde de crue.

L'enjeu n'est pas seulement régional mais aussi national. En effet, la multiplication des constructions en zones inondables tend à augmenter les dommages que les assureurs doivent rembourser en cas d'arrêté interministériel déclarant l'état de catastrophe naturelle. On rappelle que depuis la loi Tazieff, les propriétaires immobiliers (et de véhicules terrestres à moteurs) paient obligatoirement la prime cat-nat lors de la souscription de l'assurance. La prévention permet alors de maintenir la viabilité du dispositif français. La multiplication des désastres et des coûts associés aboutirait à reconsidérer le dispositif existant.

Des désastres intervenus en France (en 1999 dans l'Aude, en 2002 à Aramon, dans le Gard) ou hors de France (en 2004 à la Nouvelle Orléans) permettent de comprendre la reconnaissance du risque digue depuis le nouveau millénaire. D'où les ajustements en cours pour le prendre en compte lors des révisions des PPR, alors qu'il était souvent absent des documents antérieurs. Il est désormais reconnu par la circulaire du 30 avril 2002 et par le décret du 11 décembre 2007 relatif à la sécurité des ouvrages hydrauliques, comme 
par la doctrine inondation du Rhône. Le risque digue affiche les limites de fiabilité des ouvrages hydrauliques et vise à empêcher que les digues soient utilisées comme argument d'urbanisation au motif de l'élimination de tout futur dommage. Il défend donc des enjeux locaux et nationaux, plus explicitement. L'interdiction de construire dépend de la hauteur de l'ouvrage et des scénarios de rupture qui peuvent être envisagés. L'affichage du risque digue par l'Etat engage la responsabilité du maire, en charge par le Code général des collectivités territoriales de la sécurité, de la salubrité publique et de prévenir les dangers sur sa commune. S'agissant du Rhône, les digues de la CNR contraignent l'occupation du sol de façon uniforme : une bande digue forfaitaire de 100 mètres rend inconstructibles les terrains jouxtant les ouvrages, tant par principe de précaution que pour maintenir active la conscience d'un risque.

\subsection{Localement, la nécessité de composer avec des principes extra-locaux}

A l'échelle communale, la gestion du risque via le PPR est très marquée par les possibilités locales et par les enjeux à préserver, qui diffèrent notoirement à Culoz et à Chanaz (partie 2).

A Chanaz, le risque digue illustre un arbitrage entre la fonction correctrice/protectrice de la digue et sa contribution (involontaire) à la préparation d'un désastre. La partie constructible de la zone rouge correspond à de l'habitat palafittique (sur pilotis), qui prend en compte l'aléa d'inondation, aussi en lien avec l'inondation de la Chautagne et l'exutoire du lac du Bourget que représente le canal de Savières (Figure 2). Dans ce cas, on reconnaît alors que la réponse apportée espère limiter l'intensité des dommages sans toutefois pouvoir éliminer tout dommage. Elle permet surtout de rendre le PPR acceptable aux yeux du Conseil municipal, tout en prenant en compte les principes nationaux et enjeux économiques sous-jacents : afficher le risque d'inondation, espérer réduire l'intensité des futurs dommages, tout en les rendant compatibles avec la préservation des enjeux communaux, associés à d'autres types de risques, 
ici socio-économiques. Le risque digue est donc atténué à Chanaz. Ce serait aussi potentiellement le cas à Culoz, si le Conseil municipal suivait la possibilité offerte en 2015 par la décision préfectorale de considérer que les digues CNR, toujours en eau, s'apparentent à des barrages, supposés de fiabilité supérieure. Or, à ce jour, cela n'est pas l'option choisie à Culoz.

L'évolution des zonages des deux PPR confirme donc bien la volonté de trouver des arbitrages entre plusieurs acteurs majeurs, et agissant à des échelles différentes : l'Etat français et les sociétés de mutuelles/assurances, les préfets, la CNR, mais aussi les institutions plus locales (Plan Rhône, syndicats intercommunaux) sans oublier l'acteur majeur local, la municipalité.

Ce bilan, classique en géographie, permet d'insister sur la reconnaissance plus explicite des limites inévitables des politiques de prévention, ainsi que sur la concurrence d'enjeux multiples et simultanés, justifiant ainsi le recours à la notion de résilience. Il nous permet aussi de mieux comprendre et d'illustrer pourquoi la réflexion académique comme la gestion des risques sont passées d'une approche dite aléa centrée, à une approche reconnaissant plus la construction sociale des risques, territorialisée, avant de venir plus sur les lectures par la résilience. Ces approches successives coexistent aujourd'hui, et la comparaison des deux sites permet de l'illustrer.

\section{L'apport de la notion de résilience à la compréhension et à la gestion des risques : discussion et illustration}

\subsection{L'éclairage critique de la territorialisation des risques}

Les critiques d'une gestion des risques fondée sur un paradigme strictement moderne, qui vise la réduction des risques de désastres jusqu'à leur élimination, ne sont pas nouvelles. On peut y voir le maintien de la lecture aléa centrée, qui spatialise les risques. Cette approche tend vers le risque zéro, en espérant réduire l'intensité des futurs dommages grâce à l'ouvrage de correction (Figure 4) sans oublier que le risque zéro n'existe pas (Godard 
et ali., 2002 ; Dupuy, 2002). Dans les faits, les travaux de correction, qui agissent sur l'aléa, continuent à légitimer parfois le passage de zones rouges à des zones bleues, constructibles sous conditions. C'est ce qu'on observe à Culoz pour les risques associés aux chutes de blocs, par exemple (Figure 4). La spatialisation des risques fait référence à la seule géométrie d'une localisation, dans un repère spatial absolu, avec toutes les limites de cette lecture : elle ne permet pas de comprendre pourquoi, comme ici, il existe un traitement opposé du risque dans deux cas confrontés à un aléa d'inondation comparable. Elle entrave la prise en compte d'enjeux différents posés à l'échelle du système cat-nat et à celle des conseils communaux. Enfin, la spatialisation des risques ne permet pas de montrer les limites de l'analyse $\mathrm{du}$ risque, tout particulièrement lorsqu'elle se fait en silo, ignorant par exemple des problématiques d'emploi ou de développement territorial des communes.

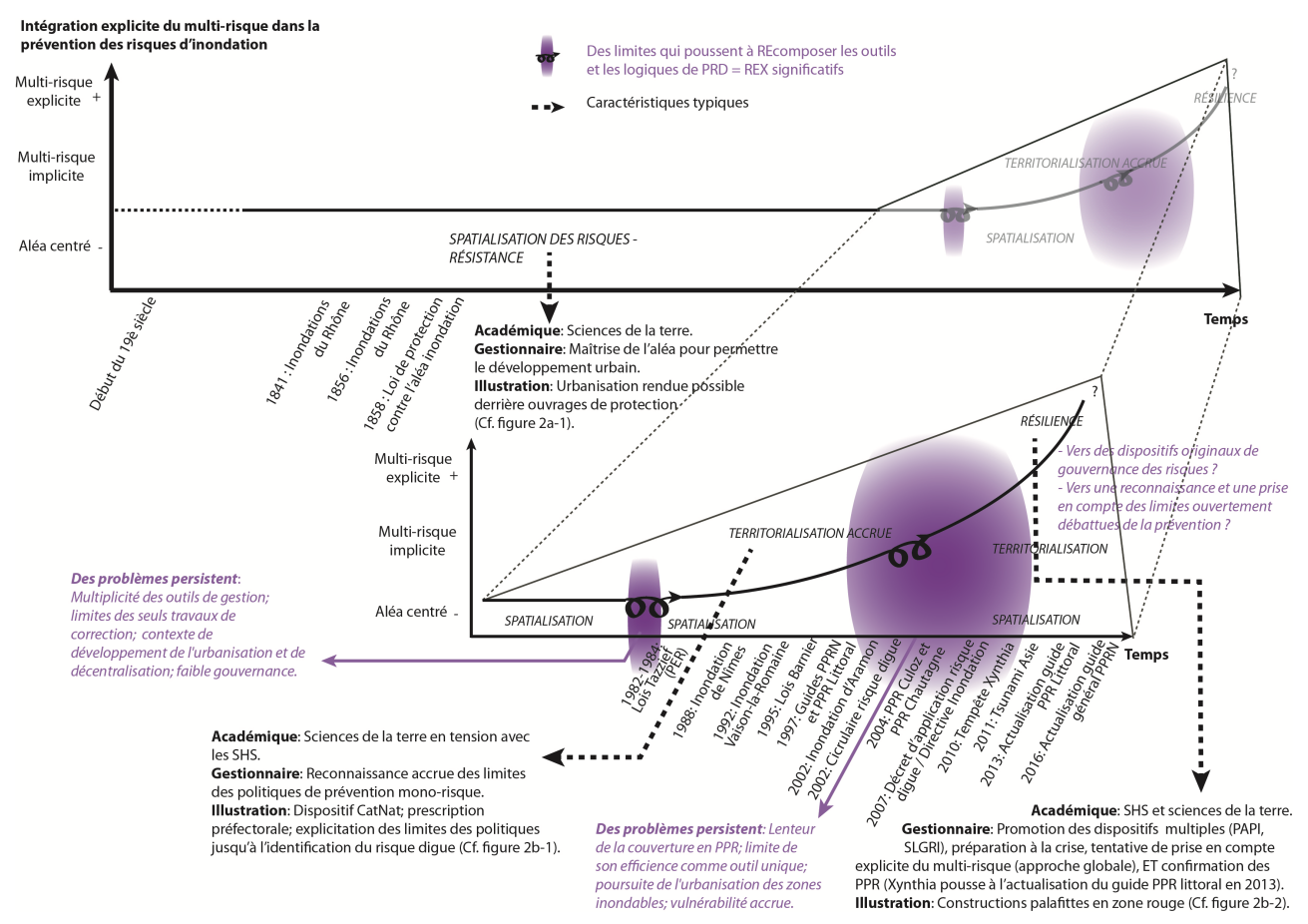

Figure. 4 : Recompositions de la prévention des désastres en Chautagne. 
Les retours d'expériences recomposent la prévention des risques de désastres et le développement local à Chanaz et à Culoz.

Un même objet (ex. la digue, qui passe d'un statut d'ouvrage de protection à un facteur de risque) traverse le temps et les différentes logiques de prévention. Au fil du temps, cet objet prend un sens et joue un rôle différent dans les politiques de prévention comme dans le développement local. Il se recompose avec les logiques de prévention des risques de désastres qu'il accompagne.

\section{Figure. 4: The reshaping of disaster prevention policies in Chautagne}

Experience returns reshape disaster risks prevention policies and local development in Chautagne.

A very same object (e.g. a dike, as a protective equipment turns into a driver of risk) goes through time and the different logics of prevention. Over time, this object takes different meanings and plays different roles in prevention policies as well as in local development. The object reshapes itself, likewise the logics of disaster risks prevention it comes with.

Ainsi, bien qu'encore structurante aujourd'hui (Figure 2), la lecture aléa centrée n'explique pas à elle seule la gestion des risques de désastre. D'autres approches tentent de l'expliquer. L'une d'entre elles, spécifiquement géographique, s'attache à défendre un degré d'intégration plus fort pour comprendre et gérer les risques, au moyen d'une entrée dite territoriale. L'approche territorialisée des risques articule ces derniers à la complexité de la notion de territoire, et se démarque alors d'une « simple » spatialisation de leur gestion, constitutive de l'approche aléa-centrée (Pigeon, 2005).

L'approche territorialisée des risques correspond, elle, à une approche plus intégrée, moins fragmentée que l'approche aléa-centrée. Elle est définie comme un prisme de lecture qui permet de comprendre et éventuellement de 
répondre aux problèmes concrets posés par la plurifactorialité des risques, par la diversité des acteurs et de leurs intérêts, mais aussi par les interférences entre les échelles spatiales et temporelles (Reghezza-Zitt, 2015 ; Meschinet de Richmond et Reghezza, 2010). La notion de territoire n'est certes pas exempte des écueils possibles d'une mise en application trop statique et descriptive, voire essentialiste. Mais les critiques de cette lecture réductrice du territoire sont légion, tant dans la littérature francophone (Jean et Calenge, 2002) qu'anglophone (Antonsich, 2011). L'approche territorialisée des risques résiste à ces écueils lorsque l'on considère qu'elle porte sur l'ensemble des processus de construction des risques. Ces processus ne se limitent pas au seul bornage, c'est-à-dire à l'analyse et à la correction de l'aléa, ni simplement à la gestion, c'est-à-dire au contrôle de l'urbanisation future et à la correction des éléments exposés inégalement vulnérables. Ces dimensions sont présentes dans les zonages des PPR. Mais on convient que tous ces processus recouvrent des aspects matériel et idéel, politique et culturel, différemment identifiés, appropriés, et représentés. Les risques s'inscrivent ainsi dans une logique de construction sociale (GarciaAcosta, 2005). Avec ce type de lecture, l'aléa identifié, ici celui lié aux inondations du Rhône, est intégré et traité en fonction des attentes et des ressources différenciées de plusieurs acteurs et territoires impliqués, à plusieurs échelles, dans la prévention de plusieurs types de risques. Cette approche multirisque, très politique, est particulièrement sensible aux effets de contexte (Rebotier, 2012a).

On peut dès lors mettre l'accent sur les freins de l'approche territorialisée pour l'action: le poids de la production d'un territoire avec toutes ses contradictions, la diversité des acteurs et de leurs intérêts, et la nécessité de prendre en compte les articulations scalaires. En termes méthodologiques, cette lecture du territoire est comptable des travaux de Lefebvre sur la production de l'espace (2000). Elle peut se traduire par une approche socio-historique sensible aux caractéristiques particulières des 
territoires, aux héritages de peuplements et à la contextualisation nécessaire des situations (Rebotier, 2012b). Mais dans les faits, la difficulté d'articuler les principes généraux de la prévention des désastres à la complexité des territoires et à l'obligation de l'action (Propeck-Zimmermann, 2015) amène la gestion à plutôt adopter les principes de la spatialisation des risques (Reghezza-Zitt, 2015). Si cette dernière présente l'avantage d'une opérationnalisation plus aisée, car plus normée, elle reste la marque d'une approche fragmentée, peu perméable aux apports intégrateurs de l'approche territorialisée des risques. Ainsi, la territorialisation est la marque de l'évolution de la mise en place de politiques de prévention plus attentives aux échelles locales et aux contextes socio-spatiaux d'essence complexe. Néanmoins, elle porte aussi les limites de sa capacité à valoriser ses apports, à la fois spatiaux et géopolitiques, du fait même de leur complexité.

C'est ce qu'illustre le PPR en tant qu'outil de gestion des risques. Il constitue certes une avancée au regard de l'approche aléa-centrée, car il est question d'enjeux pluriels sur le territoire : la plurifactorialité des risques et la multiplicité des acteurs appellent des arbitrages et sont à l'origine de négociations intenses pour les définir (Liévois, 1998). Mais cette avancée n'est que partielle. La prise en compte de l'approche intégrée que requiert la prévention des risques de désastre n'est qu'implicite: l'outil PPR n'explicite pas assez l'existence de plusieurs types de risque. Il ne parvient pas à les articuler clairement y compris dans ses applications multi-aléas. En outre, si le processus réglementaire d'élaboration du PPR invite clairement les parties prenantes à la concertation, le principe même d'intégration n'y trouve pas de traduction concrète. Dès lors, lorsqu'une transaction intervient, aboutissant à aménager ou rendre constructibles des terrains en zone à risques en contrepartie d'une réduction de vulnérabilité à une échelle plus globale, les termes de cette transaction ne sont pas fixés, ni explicités. Enfin, le PPR ouvre sur une faible gouvernance des risques, une fois approuvé. 
Face au demi-échec (ou à la réussite partielle !) de la territorialisation à développer une gestion intégrée des risques, on peut faire l'hypothèse que l'approche par la résilience peut offrir une nouvelle avancée pour la compréhension et pour la gestion. La résilience, elle aussi, met l'accent sur la dimension politique des risques, la nécessaire contextualisation, la présence d'enjeux multiples et d'acteurs aux intérêts contradictoires. Mais cette dernière approche présente deux apports potentiels: rendre plus explicites les contradictions et les limites de la prévention des désastres ; et offrir un support à une gouvernance plus intégratrice. La résilience peut-elle être à la hauteur de ces attentes d'intégration de la plurifactorialité des risques et d'articulation explicite des contradictions de leur gestion?

\subsection{La dimension politique de la prévention des risques de désastres}

De fait, considérer la résilience à Chanaz et à Culoz comme le résultat de la capacité à élaborer des solutions à la fois nécessaires et nécessairement limitées, qui font sens pour une majorité d'acteurs, permet d'articuler logiquement quelques-uns des aspects contradictoires et problématiques que recouvre ici le risque d'inondation officiellement identifié. Cette conception de la résilience permet de prendre en compte l'existence d'autres types de risques. Gérer le risque d'inondation ne peut se comprendre ni se faire sans recourir à une forme de transversalité, sans intégrer les risques liés à l'emploi (activités liées à la plaisance fluviale à Chanaz, à la CIAT à Culoz), les effets induits sur les valeurs foncières, les pertes possibles que les propriétaires, mais aussi les assureurs, devraient compenser en cas de

survenue d'une inondation. Le risque est donc fondamentalement et doublement affaire politique (Pigeon, 2005). Non seulement il appelle des décisions qui reposent sur des valeurs, ou des choix de société et pas seulement sur l'application d'une norme; mais il est aussi le reflet d'une diversité d'acteurs, aux intérêts propres, qui doivent composer ensemble et dans la durée selon des arrangements aux contours fluctuants. 
Le fait d'évoquer une dimension politique comme un attribut de la résilience permet de revenir sur les choix, les arbitrages, et les rapports de force dans la négociation et l'élaboration des politiques de prévention des désastres. Dans l'idéal, la défiance cèderait alors la place à la confiance. De manière symptomatique, des travaux récents insistent sur le caractère fondamentalement politique de la résilience (Rufat, 2015). Ceci contribue tant à une meilleure compréhension des risques qu'à envisager l'émergence des pistes concrètes et plus explicites pour leur gestion. Par la résilience face au risque d'inondation, il n'est plus seulement question de protéger, ou de réduire des impacts attendus en mobilisant de façon optimale des ressources techniques ou règlementaires. Il s'agit d'être en capacité de produire la moins mauvaise des solutions pour le plus grand nombre d'acteurs, à l'échelle du territoire d'étude, mais bien évidemment aussi, au-delà. Et cette capacité passe par la possibilité (qu'offre la résilience ?) de représenter plus explicitement et de prendre en compte, dans la compréhension et dans l'action, les limites nécessaires des politiques de prévention, multirisques.

\subsection{La résilience, une clé pour dépasser les limites de l'approche territorialisée des risques de désastres?}

Malgré une lecture dynamique de l'espace comme produit social, l'analyse que permet de livrer l'approche territorialisée des risques peut vite s'enfermer dans une lecture politique, voire biopolitique, ne voyant plus dans le risque qu'une forme de médiation entre groupes d'acteurs aux rapports de pouvoir asymétriques (Rebotier, 2011). Si cette lecture très foucaldienne n'est pas inutile, et correspond à une partie de ce que sont les risques dans le monde social, elle ne permet pas de faire émerger trois éléments clés que le prisme de la résilience peut, lui, rendre plus visibles. Il le peut notamment sur la base d'une définition de la résilience qui consiste en la capacité à élaborer une solution partagée de prévention des désastres admissible par le plus grand nombre de parties prenantes et qui les implique 
dans sa mise en œuvre. Tout en admettant que cette réponse ne permet pas d'éliminer tout dommage, voire contribue à les produire.

- Notre définition de la résilience rend centrale la tension suscitée par les standards de prévention nécessaires et tout à la fois imparfaits. La discussion inévitable des limites et les arbitrages qui accompagnent souvent ces mesures amènent à travailler à une gouvernance des risques, à trouver des dispositifs pour faire du lien, là où la territorialisation des risques, à travers la déclinaison locale des politiques de prévention, peut amener (mais n'amène pas forcément !) à opposer des groupes d'acteurs. L'enjeu de la reconnaissance et de l'affichage plus explicite de cette tension, et des façons d'y apporter des réponses, pose la première pierre d'un travail plus proprement stratégique de la gestion des risques, auquel la territorialisation des risques semble moins inviter. De cette ouverture de la résilience à une dimension plus politique/stratégique que politique/partisane débouchent les points suivants, l'un plus conceptuel et l'autre plus appliqué.

- Le caractère plastique de la résilience, malgré ses limites, permet de poser les problèmes de façon plus transversale et moins spécialisée qu'à l'habitude. Il permet notamment de faire tenir, en cohérence, un traitement différencié du risque d'inondation par les mêmes acteurs, sur deux sites très proches, alors que nous avons vu combien les différences d'aléa, à ellesseules, ne peuvent expliquer ces spécificités de traitement. La recherche de résilience peut incorporer des tensions, voire des contradictions que l'on trouve sur le terrain, et même leur donner du sens... sans en chercher nécessairement des responsables, ni des coupables, comme on pourrait le faire plus probablement dans le cadre d'une approche territorialisée des risques. L'ouverture que permet la résilience dans la façon de penser les choses peut être mise à profit (Chandler, 2014), comme un outil ou un support conceptuel. Mais ce n'est là, bien sûr, qu'une possibilité.

- Dans un registre plus opérationnel, la résilience peut apparaître comme la recherche d'un instrument pédagogique capable de mettre autour de la table 
des acteurs a priori peu liés entre eux, sur le territoire, ou dans la gestion d'un espace, mais qui le sont pourtant, dans les faits (Reghezza-Zitt et Rufat, 2015b). Il s'agit d'en faire des acteurs de la réponse apportée dans sa mise en œuvre même. On pense notamment aux acteurs communaux, porteurs d'enjeux locaux, aux représentants locaux de l'Etat ou aux acteurs régionaux, tels que la CNR qui intervient sur chacun des sites.

La recherche de résilience peut établir une ligne d'action cohérente, défendable, pour tous, et applicable sur différents sites, plus que la négociation d'outils de gestion tels que les PPR, sans pour autant faire obstacle à leur mise en œuvre. Cette vocation à faire collaborer les parties prenantes dans la mise en œuvre de la solution retenue tend à rompre avec une application rigoriste du cadre moderne (fonctionnel, spécialisé, linéaire). Il s'agit moins de rejeter ce cadre que d'exploiter la créativité qu'il offre pour l'adapter à son environnement complexe et le rendre du mieux possible opérant. Dans les faits, les outils de gestion actuels incorporent les phases de gestion et de compréhension antérieures sans les éliminer (Figure 4). La recherche de résilience permet de ne pas considérer les limites des outils existants (par exemple, les zones rouges des PPR) comme des obstacles insurmontables, mais fondamentalement comme des limites à intégrer aux choix des acteurs, et à afficher explicitement au sein des logiques de peuplement. Selon les mêmes principes, les limites que représentent les zones rouges de Culoz et de Chanaz sont traitées différemment. Leur affichage est à l'avenant. La recherche de résilience permet de faire cohabiter les limites introduites par les outils de gestion (zones rouges) et leur incorporation opposée aux logiques de peuplement (secteur inconstructible $V s$ constructions palafittiques).

Ainsi, la résilience permet au moins tout autant que l'approche territorialisée des risques de comprendre la plurifactorialité des risques de désastres et la multiplicité des enjeux qu'ils impliquent. Les différences entre la genèse, le traitement et l'affichage des risques liés au Rhône à Culoz et à Chanaz, 
présentées précédemment, en sont une illustration. Dans la même ligne, il est également possible de comprendre les contradictions qui sous-tendent les risques, tout comme la diversité des parties prenantes et de leurs intérêts, et ce à plusieurs échelles. Mais la résilience semble présenter des aspects plus pragmatiques, une plus grande facilité à circuler entre (et à faire dialoguer) les registres académiques et opérationnels... sans pour autant apporter la garantie de cette circulation ni des retombées attendues dans le registre de l'action. Il appartient à ceux qui s'en emparent de jouer une partition délicate, entre deux grands pôles. D'un côté, on a le travail d'analyse critique, desservi par la dénonciation à l'excès. D'un autre, on a l'obligation d'opérationnalisation qui sacrifie souvent à cette obligation le retour critique sur les choix de l'action.

Sans y parvenir totalement, la résilience permet de mieux comprendre les politiques de prévention des désastres (voire de les orienter ?) comme dans le cas de constructions rendues possibles dans le PLU de Chanaz sur des zones rouges du PPR.

\subsection{Des apports toujours politiquement contingents : le cas des palafittes dans la zone rouge du PPR de Chanaz.}

Notre lecture est marquée par une définition de la résilience plus orientée vers la gouvernance. Mais elle n'en occulte pas les aspects plus techniques et appliqués. Le caractère le plus original, et potentiellement prometteur, consiste notamment à faire de la discussion explicite des limites des politiques de prévention une arène tout à la fois technique et politique.

La résilience ne vise en aucun cas à neutraliser les rapports de force, ni ne cherche à mettre en cause certains acteurs plutôt que d'autres. Appliquée au risque, elle semble moins marquée que l'approche territorialisée par la recherche de dénonciation, voire de correction de rapports asymétriques, plus en lien avec une lecture radicale. Certes ainsi plus opératoire, c'est aussi pour cela qu'elle est fréquemment dénoncée comme naturalisant les politiques de prévention des désastres. C'est le contenu programmatique 
libéral et peu transformateur que la résilience recouvrirait dans le domaine de la gestion des risques, qui est ici visé.

Les deux terrains mobilisés par cet article illustrent ces différents aspects. En effet, l'abandon du projet de port de plaisance fluviale par la municipalité de Culoz ne peut en aucun cas s'expliquer par le seul risque d'inondation, tel qu'il est présenté par le zonage du PPR. Ce serait méconnaître les enjeux principaux de la commune (la voie ferrée, la CIAT), qui ne sont pas liés au fleuve, et ignorer l'existence de ressources foncières. Dans ces conditions, il est plus facile de conserver la zone d'expansion des crues du Rhône afin de renforcer la protection de Lyon par les digues, à l'aval, mais aussi plus largement de préserver le système national cat-nat et les sociétés d'assurance partenaires de la prévention. La résilience est donc bien ici la recherche d'une conciliation entre plusieurs types de risques. Localement, elle se traduit par des terrains en friche, par la renonciation à utiliser les anciennes gravières, ou à tirer parti des aménagements du Rhône, aussi fiables puissent-ils être, comme c'est le cas des digues CNR.

En revanche et de façon contrastée, à Chanaz, la recherche de résilience se matérialise par l'urbanisation rendue possible, certes limitée et adaptée, de terrains explicitement associés au risque d'inondation : pour la commune, il $\mathrm{y}$ aurait plus de risques collectifs à renoncer à valoriser le port de plaisance fluviale. Ces risques sont exacerbés par le passé de la commune, attachée à la valorisation économique du transport par voie d'eau (le Rhône, le canal de Savières), mais aussi à la valorisation foncière des terrains proches de ces voies. Une solution de conciliation a été trouvée avec l'État sans que celuici ait eu à renoncer à la prévention. Elle passe par l'assouplissement local du risque digue, et plus encore par l'affichage d'un zonage rouge du PPR ponctuellement très atypique. Ce dernier n'est plus incompatible avec la présence, même limitée, de logements. Le règlement du PPR précise « des zones rouges dites inconstructibles $(\mathrm{R}, \mathrm{RD}, \mathrm{RH})$ : certains aménagements peuvent toutefois y être autorisés, assortis d'une prise en compte du risque, 
mais la vocation de ces zones est globalement le maintien du bâti à l'existant ». Le zonage confirme aussi que les palafittes se trouvent bien en zone rouge, malgré la tendance à représenter ce secteur comme un ancien cours du Rhône, en bleu clair (Figure 2).

En délibération, le Conseil municipal de Chanaz se félicite le 19 décembre 2014 de l'accord trouvé : « le développement touristique de notre commune reste possible dans le secteur du port pour la construction d'habitations sur pilotis [...]. De plus, le règlement présenté permet la réalisation de travaux d'extension de bâtiments existants en zone rouge sous certaines conditions, ce qui n'est pas possible actuellement». Ce texte est antérieur à l'approbation du PPR de 2015, et se réfère au zonage associé au PPR de 2004. Dans sa délibération du 16 janvier 2015, le Conseil municipal « décide d'amortir à partir du $1^{\text {er }}$ janvier 2015 sur une durée de 25 ans le montant total des travaux des chalets sur pilotis et des aménagements portuaires de la base lacustre $»$.

Dans ces conditions, la résilience comme résultat de l'action sur le territoire de Chanaz se présente sous une forme apparemment très différente de celle que l'on trouve à Culoz. Mais elle correspond bien aussi à l'explicitation des limites des ouvrages de correction, avec la zone rouge du PPR, et elle explicite aussi les enjeux municipaux spécifiques. Il s'agit bien d'une recherche de solution, d'un processus, aux résultats très temporaires, et précaires, qui implique directement la commune dans la mise en œuvre de mesures à sa charge. La solution trouvée n'interdit pas les projets de construction, à enjeux locaux forts, qui accompagnent la valorisation du port de plaisance. Les bâtiments admis sont limités, et intègrent aussi le risque d'inondation: la solution trouvée permet aussi de répondre aux enjeux régionaux, et nationaux. Mais cette solution ne permet pas d'éliminer tout dommage, ce qu'affiche explicitement tant le zonage du PPR que le règlement qui l'accompagne. 


\section{Conclusion}

La comparaison entre les sites de Chanaz et de Culoz permet d'illustrer une gradation en trois stades de la prévention des risques de désastres. Dans les deux communes, cette gradation est identifiable, tant sur les plans d'aménagement que sur le terrain. Dans chacune, elle passe par le contrôle recherché de l'aléa hydraulique puis des tentatives de territorialisation des risques liés aux inondations, retranscrites ici dans les zonages du PPR. Ces deux recherches de solutions se combinent plus qu'elles ne se succèdent. Les contradictions que la comparaison met en évidence ne conduisent pas à récuser l'une ou l'autre de ces approches mais offrent une ouverture vers la résilience. Ce troisième stade permet d'intégrer les limites des phases d'aménagement précédentes en mettant en avant de nouvelles réponses pragmatiques, parfois d'aspect singulier, sinon contradictoire (le risque digue, construire en zone rouge du PPR), qui contribuent à recomposer ces phases d'aménagement.

Mais aucune intégration ne produit de solution définitive.

Intégrer différentes démarches dont les limites ont été reconnues a un prix sur le plan académique : la polysémie ou les contradictions que révèle tout particulièrement le risque digue. L'ouvrage de correction de l'aléa et de protection des biens et personnes exposés devient potentiellement un ouvrage qui contribue à préparer un désastre, même sans le vouloir.

Intégrer différentes démarches dont les limites ont été reconnues a aussi un prix pour les gestionnaires et les concepteurs des politiques de prévention des désastres : expliciter les limites des politiques antérieures peut favoriser les conflits autour des politiques de prévention. Ces dernières apparaissent centrées sur un type de risque, ici, celui lié à l'aléa inondation, alors qu'un tel centrage ne permet pas d'éliminer tout dommage. Il faut donc expliciter non seulement les limites des politiques antérieures, mais encore les arbitrages entre les différents acteurs qui en sont parties prenantes, à différentes échelles. Les zonages des PPR suggèrent ces arbitrages, mais ils 
les explicitent très mal. La politique de prévention des risques de désastres, tout comme son pan académique, deviennent alors plus explicitement multirisques. L'exemple du port de plaisance de Chanaz confirme les impasses $\mathrm{du}$ raisonnement académique comme de la décision et de la gestion lorsqu'ils sont explicitement centrés sur un seul type de risque. Le prix à payer pour plus de cohérence est celui de l'affichage du multirisque et de la recherche d'une réponse globale, conciliant les principaux intérêts en présence. La notion de résilience concourt à ce résultat dès lors qu'elle permet d'élargir la gamme des réponses à apporter à l'aléa inondation pour lequel la question de la prévention est ici posée.

Intégrer différentes démarches dont les limites ont été reconnues a aussi des avantages. Cela permet d'expliciter pourquoi il est nécessaire d'intégrer plusieurs approches et plusieurs types d'acteurs, comme plusieurs types de risques, dans la compréhension, dans la conception et la gestion des politiques de prévention des désastres. On peut retrouver ici la tendance mondiale à valoriser les systèmes de gestion de la connaissance, qui, tout comme la résilience, ont aussi le vent en poupe depuis le début du $21^{\text {ème }}$ siècle. L'un de ces systèmes de gestion de la connaissance est représenté, en France, par l'observatoire national des risques naturels, né en 2012, principalement après les désastres associés aux inondations du Var et du littoral atlantique, en 2010. Leur principe est le partage et l'intégration de bases de données sur les risques, venant d'institutions variées, afin d'affiner les diagnostics sur les politiques de prévention des désastres, principalement à échelle supra-locale. La question posée reste celle de la transcription de tels systèmes à l'échelle locale.

Mais l'autre défi majeur de l'intégration des limites des politiques antérieures est d'expliciter ces limites pour mettre en œuvre des mesures additionnelles, notamment pour la sécurité des personnes à l'échelle locale. La présence de logements en zone rouge du PPR de Chanaz et d'un règlement qui en autorise l'aménagement peut illustrer la notion de 
résilience, dans la mesure où les avantages et surtout les limites (pour qui ?) des politiques sont plus clairement explicitées. N'oublions pas que le risque digue était affiché sur le plan de prévention des risques de La Faute-sur-Mer, en 2007, trois ans avant le désastre de février 2010 et ses 29 morts mais que les conséquences n'en avaient pas été tirées en matière de mesures additionnelles (Pigeon, 2012b).

De ce fait, nous proposons de définir la résilience comme le résultat d'un processus précaire (Figure 4) qui vise à développer des politiques de prévention et des formes de peuplement les moins mauvaises pour le plus grand nombre de parties prenantes, à différentes échelles. Ces solutions ne permettent pas d'éliminer tout dommage, voire contribuent à les produire. De telles limites invitent à impliquer plus explicitement les différents acteurs dans l'élaboration de ces solutions. A Chanaz, les palafittes en zone rouge du PPR nous semblent illustrer pleinement la recherche de résilience, ainsi définie, au même titre que les friches qui occupent le site envisagé pour le port de plaisance à Culoz, car ce dernier ne sera pas réalisé, comme le confirme l'enquête menée auprès de la mairie en décembre 2016.

\section{Bibliographie}

Alexander, D. (2013), «Resilience and disaster risk reduction: an etymological journey », Natural Hazards Earth System Sciences, $\mathrm{n}^{\circ} 13$, p. 2707-2716.

Antonsich, M. (2011), «Rethinking Territory», Progress in Human Geography, $\mathrm{n}^{\circ} 35$ (3), p. 422-425.

Birkmann, J. (2006), Measuring vulnerability to natural hazards. Towards disaster resilient societies, Tokyo, United Nations University press, 720 p. Bravard, J-P. (1986), Le Rhône, du Léman à Lyon, Lyon, La Manufacture, $451 \mathrm{p}$. 
Brun, A. et Gache, F. (2013), « Risque inondation dans le Grand Paris : la résilience est-elle un concept opératoire ?», $\operatorname{VertigO}$, http://vertigo.revues.org/14339

Chandler, D. (2014), Resilience. The Governance of Complexity, Londres et New York, Routledge, 268 p.

Combes, H. (2007), La ville endormie ? Le risque inondation à Lyon. Thèse de doctorat en géographie. Université Lumière Lyon 2.

Donze, J. (2007), «Risque : de la recherche à la gestion territorialisée », Géocarrefour, n 82 (1-2), https://geocarrefour.revues.org/1395

Dupuy, J-P. (2002), Pour un catastrophisme éclairé. Quand l'impossible est certain, Paris, Seuil, 224 p.

Evans, B. et Reid, J. (2014), Resilient Life. The Art of Living Dangerously, Cambridge, Polity Press, 208 p.

García Acosta, V. (2005), «El riesgo como construcción social y la construcción social de riesgos ", Desacatos, $\mathrm{n}^{\circ}$ 19, p. 11-24.

Godard, O., Henry, C., Lagadec, P. et Michel-Kerjan, E. (2002), Traité des nouveaux risques, Paris, Gallimard, $620 \mathrm{p}$.

Guézo, B. et Pigeon, P. (2015), « Les défis liés à la prévention des désastres dans les aires métropolitaines : exemple de Givors dans l'aire métropolitaine lyonnaise (France) », VertigO, http://vertigo.revues.org/15842

Gunderson, L. et Holling, C.S. (2002), Panarchy. Understanding Transformations in Human and Natural Systems, Washington, Island Press, $448 \mathrm{p}$.

Jean, Y. et Calenge, C. (2002), Lire les territoires, Tours, MSH, 300 p.

Klingeman, P.C., Bravard, J-P. et Giuliani, Y. (1994), «Les impacts morphodynamiques sur un cours d'eau soumis à un aménagement hydroélectrique à dérivation : le Rhône en Chautagne, France », Revue de géographie de Lyon, $\mathrm{n}^{\circ} 69$ (1), p. 73-87.

Lefebvre, H. (2000 [1974]), La production de l'espace, Paris, Anthropos, $485 \mathrm{p}$. 
Liévois, J. (1998), "Appropriation des risques transcrits», Revue de géographie alpine, $\mathrm{n}^{\circ} 86$ (2), p. 91-99.

Meschinet de Richemond, N. et Reghezza, M. (2010), «La gestion du risque en France : contre ou avec le territoire?», Annales de Géographie, $\mathrm{n}^{\circ} 673$, p. $248-267$.

Pigeon, P. (2014), « Risque digue: une justification à la relecture systémique et géopolitique des risques environnementaux», L'espace politique, $\mathrm{n}^{\circ} 24$ (3), https://espacepolitique.revues.org/3256

Pigeon, P. (2012a), Paradoxe de l'urbanisation. Pourquoi les catastrophes n'empêchent-elles pas l'urbanisation?, Paris, L'Harmattan, 278 p.

Pigeon, P. (2012b), «Apports de la résilience à la géographie des risques : l'exemple de La Faute-sur-Mer (Vendée, France)», VertigO, http://vertigo.revues.org/12031

Pigeon, P. (2005), Géographie critique des risques, Paris, Economica, $220 \mathrm{p}$.

Pigeon, P. et Rebotier, J. (2016), Disaster Prevention Policies. A Challenging and Critical Outlook, Londres, ISTE Press - Elsevier, 240 p. PLU de Culoz (2009), Rapport de présentation du PLU approuvé par délibération du Conseil municipal le 21 avril 2009, Mairie de Culoz, 170 p. PPR de Culoz (2004) Rapport de présentation, Mairie de Culoz, 54 p. Propeck-Zimmermann, E. (2015), Modélisation des risques et décision territoriale. Recherches sur les risques socio-techniques en milieu urbain, Mémoire d'HDR en géographie, Université de Caen Normandie.

Rebotier, J. (2012a). « Une approche territoriale des risques. Un outil pour le chercheur, critique et réflexif », Géographie et Cultures, n 81, p. 77-90.

Rebotier, J. (2012b), «Vulnerability Conditions and Risk Representations in Latin-America: Framing the Territorializing Urban Risk», Global Environmental Change, $\mathrm{n}^{\circ} 22$ (2), p. 391-398.

Rebotier, J. (2011). « Habiter les territoires et construire les risques, entre empreintes spatiales et logiques sociales - Caracas », dans Habiter les 
territoires à risque, November, V., Penelas, M. et Viot, P. Lausanne, Presses Polytechniques et Universitaires Romandes, p. 81-100.

Reghezza, M. (2015), De l'avènement du monde à celui de la planète : le basculement de la société du risque à la société de l'incertitude, Mémoire d'HDR en géographie, Paris 1 - Panthéon Sorbonne.

Reghezza-Zitt, M. (2015), « Territorialiser ou ne pas territorialiser le risque et l'incertitude. La gestion territorialisée à l'épreuve du risque d'inondation en Île-de-France », L'espace politique, $\quad \mathrm{n}^{\circ} 26 \quad$ (2), https://espacepolitique.revues.org/3543

Reghezza-Zitt M. et Rufat, S. (2015a), Résiliences. Sociétés et territoires face à l'incertitude, aux risques et aux catastrophes, Croydon, ISTE Press, $226 \mathrm{p}$.

Reghezza-Zitt, M. et Rufat, S. (2015b), « L'adaptation en Île-de-France entre injonction et recyclage. Techniques et politiques de la société de l'incertitude », Développement durable et territoires, $\mathrm{n}^{\circ} 6$ (3), http://developpementdurable.revues.org/11035

Rufat, S. (2015), «Critique de la résilience pure», dans Résiliences. Sociétés et territoires face à l'incertitude, aux risques et aux catastrophes, Reghezza-Zitt, M. et Rufat, S. Croydon, ISTE Press, p. 187-209.

Trapatel, G. et Fruchart, F. (2012), «Digues déversantes et déversoirs de l'aménagement CNR du Rhône" dans Les déversoirs sur les digues fluviales, Degoutte, G., Versailles, Quae, p. 161-166.

UNISDR (2015), Global Assessment Report on disaster risk reduction. Making development sustainable: The future of disaster risk management, Geneva, UNISDR, https://www.unisdr.org/we/inform/publications/42809 